\title{
Active learning and element-embedding approach in neural networks for infinite-layer versus perovskite oxides
}

\author{
Armin Sahinovic and Benjamin Geisler (1) \\ Department of Physics, Universität Duisburg-Essen, Lotharstr. 1, 47057 Duisburg, Germany
}

(Received 6 April 2021; revised 1 October 2021; accepted 13 October 2021; published 10 November 2021)

\begin{abstract}
Combining density functional theory simulations and active learning of neural networks, we explore formation energies of oxygen vacancy layers, lattice parameters, and their statistical correlations in infinite-layer versus perovskite oxides across the periodic table, and place the superconducting nickelate and cuprate families in a comprehensive context. We show that neural networks are capable of predicting these observables with high precision, using only $30-50 \%$ of the data for training. Element embedding autonomously identifies concepts of chemical similarity between the individual elements that are in line with human knowledge. We demonstrate that active learning efficiently composes the training set by an optimal strategy without a priori knowledge, based on the fundamental concepts of entropy and information, and provides systematic control over the prediction accuracy. This offers key ingredients to considerably accelerate scans of large parameter spaces and exemplifies how artificial intelligence may assist on the quantum scale in finding novel materials with optimized properties.
\end{abstract}

DOI: 10.1103/PhysRevResearch.3.L042022

\section{INTRODUCTION}

Over the last years, artificial intelligence (AI) algorithms have attracted increasing attention in computational materials science. Machine learning techniques [1-10] such as deep learning [11-14] allow for a variety of different intriguing and often unconventional approaches, ranging from applications in molecular dynamics [15], the unsupervised identification of latent knowledge in scientific literature [16], to the understanding of chemical trends from materials data $[17,18]$. In parallel, the increasing computational resources have driven high-throughput searches to identify novel materials with enhanced properties, which resulted in the emergence of different materials databases [19-22]. However, screening large parameter spaces by quantum-scale materials simulations, e.g., employing density functional theory (DFT), is still impeded by a high energy and time consumption.

Aiming for a more efficient strategy, here we complement systematic first-principles simulations across the periodic table with deep learning of artificial neural networks (NNs). We provide a broader perspective on the topical infinite-layer oxides (IL, $A B \mathrm{O}_{2}$ ) [23-40] and the respective perovskites $\left(\mathrm{P}, A B \mathrm{O}_{3}\right)$, discuss their statistical correlations and chemical trends, and highlight a selection of interesting compounds beyond the nickelate family. Subsequently, we show on the basis of this data that NNs are capable of understanding the formation energies of oxygen vacancy layers, as well as

\footnotetext{
*benjamin.geisler@uni-due.de

Published by the American Physical Society under the terms of the Creative Commons Attribution 4.0 International license. Further distribution of this work must maintain attribution to the author $(s)$ and the published article's title, journal citation, and DOI.
}

the lattice parameters of the individual compounds. These observables act as a fingerprint of the reduction reaction, containing, for instance, the essence of symmetry breaking, fourfold versus sixfold $B$-site coordination, the redistribution of electrons released by the oxygen vacancies, the different orbital sequence due to changes of the crystal field, and the modified hybridization. Hence, despite the complexity of these two materials classes and their relations, as evidenced by detailed statistical analysis, NNs autonomously unravel the systematics of their quantum-chemical bonding by using just $30-50 \%$ of the data for training. Subsequently, they predict the properties of all compounds, even those they have never seen, with high accuracy, well within the error bars of DFT itself. Interestingly, it turns out to be sufficient to only provide the $A$ - and $B$-site element names as input to the NNs, and no further atomic properties. Element embedding [17,41] is demonstrated to establish a very unique AI understanding of the chemical relations between the individual elements that mirrors the conventional picture of the periodic table. Finally, we show that combining these techniques with active learning $[2,42]$ allows for an efficient screening of the materials parameter space, being clearly superior to a randomly selected training set and offering systematic accuracy control. We provide detailed visual insight into the algorithm's working mechanisms and its performance, exemplifying the potential of AI to considerably accelerate high-throughput materials optimization.

\section{METHODOLOGY}

We performed first-principles simulations in the framework of DFT [43] as implemented in the Vienna $a b$ initio simulation package $[44,45]$ using the generalized gradient approximation as parametrized by Perdew, Burke, and Ernzerhof [46] to construct a database of ground-state energies 

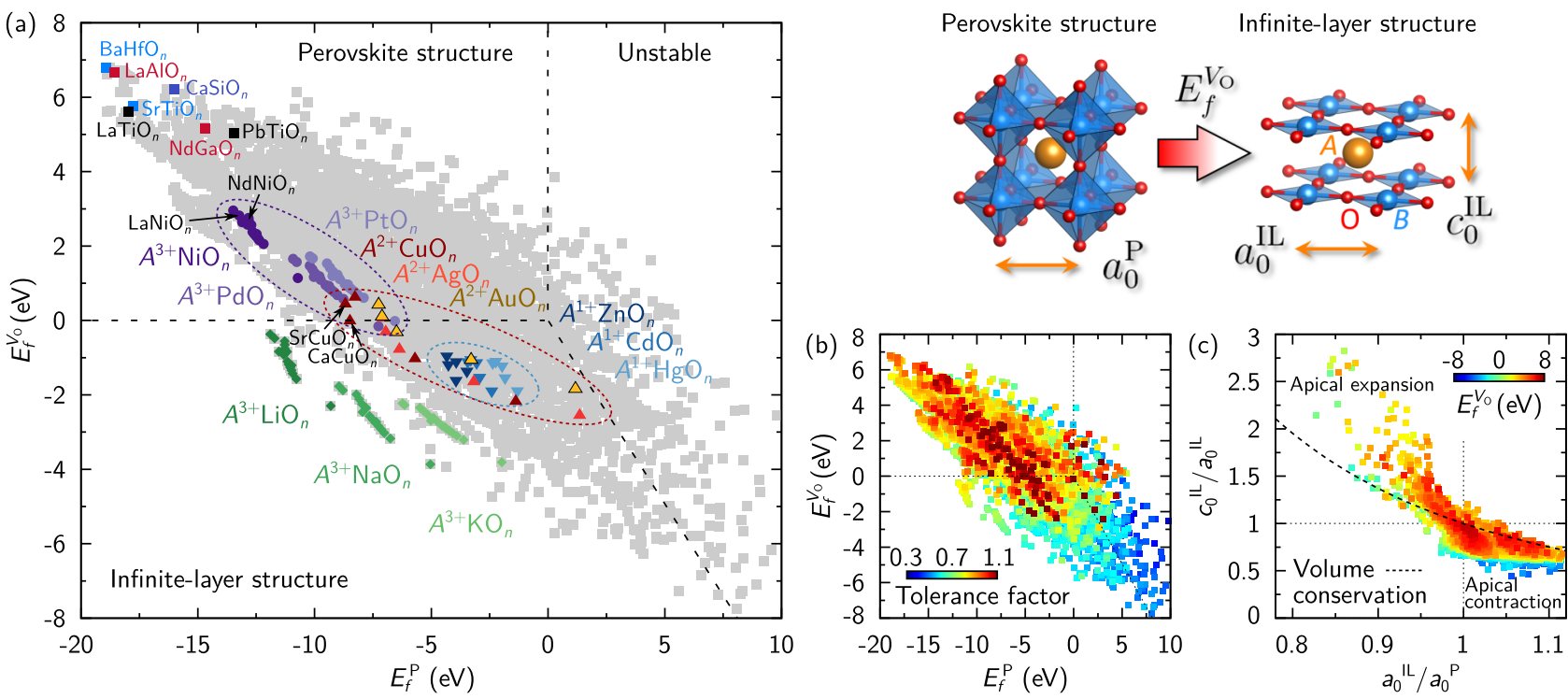

FIG. 1. (a) Reduction of the apical oxygen ions in perovskite $(\mathrm{P})$ oxides $(n=3)$ results in the emergence of the anisotropic infinite-layer (IL) structure $(n=2)$. This reaction is associated with the energy $E_{f}^{V_{\mathrm{O}}}$. The phase diagram compares the relative stability of the IL versus the $\mathrm{P}$ structure as a function of the respective $\mathrm{P}$ heat of formation for the entire data set. A number of interesting compounds is highlighted, together with a selection of technologically important materials as reference points. (b) Superposition of the diagram in (a) with the Goldschmidt tolerance factor $t$. (c) Structural perspective on the data, comparing apical to basal changes upon reduction and superimposing them with $E_{f}^{V_{\mathrm{O}}}$.

and optimized lattice parameters for 4692 combinations of different elements at the $A$ and $B$ sites (as detailed below) for both the perovskite (P) and the infinite-layer (IL) oxides, which were modeled by using cubic [8] and tetragonal [28,30] unit cells, respectively. We largely adopted the DFT $+U$ standards [47] of the Materials Project database [19,48,49] and used their ground-state crystal structures for the elemental bulk references. NNs were realized in Keras/Tensorflow 2 [50,51], and the active-learning algorithm was developed in Python 3. The formation energies of the oxygen vacancy layers are determined from DFT ground-state energies by $E_{f}^{V_{\mathrm{O}}}=E\left(A B \mathrm{O}_{2}\right)-E\left(A B \mathrm{O}_{3}\right)+\mu_{\mathrm{O}}$, where $\mu_{\mathrm{O}}=\frac{1}{2} E\left(\mathrm{O}_{2}\right)$ models the oxygen-rich limit [52]. The heats of formation of the $\mathrm{P}$ phase from the constituent bulk elements read $E_{f}^{\mathrm{P}}=E\left(A B \mathrm{O}_{3}\right)-E(A$ bulk $)-E(B$ bulk $)-3 \mu_{\mathrm{O}}$. An analogous quantity $E_{f}^{\mathrm{IL}}$ can be defined for the IL phase. All energies are given per formula unit.

\section{DATA EXPLORATION AND STATISTICAL ANALYSIS}

We begin by providing an overview of the data set from a thermodynamic, a structural, and a statistical perspective. Figure 1(a) displays the entire data in a $E_{f}^{V_{\mathrm{O}}}$ versus $E_{f}^{\mathrm{P}}$ phase diagram, comparing the relative stability of the IL and the $\mathrm{P}$ structure, as well as their stability with respect to the constituent bulk elements. This is motivated by recent experiments on IL oxides that attracted considerable attention, specifically superconducting nickelates [23,29,35], which are initially stabilized as $\mathrm{P}$ films on $\mathrm{SrTiO}_{3}(001)$ via heteroepitaxy, followed by a topotactic reduction of the apical oxygen ions. $E_{f}^{V_{\mathrm{O}}}$ ranges from -8 to $+7 \mathrm{eV}$, while $E_{f}^{\mathrm{P}}$ covers almost $30 \mathrm{eV}$. The plot reveals an overall linear trend in the data, correlating the $\mathrm{P}$ stability and its reduction energy. However, the data scatters broadly around the regression line $E_{f}^{V_{\mathrm{O}}}=$
$-0.36 E_{f}^{\mathrm{P}}-1.37 \mathrm{eV}$. Superimposing this plot with the Goldschmidt tolerance factor $t=\frac{r_{A}+r_{O}}{\sqrt{2}\left(r_{B}+r_{\mathrm{O}}\right)}$ calculated from the ionic radii [Fig. 1(b)] reflects that the $\mathrm{P}$ stability (moving from right to left) increases with $t$, reducing again for $t>1$. Again, we find that the data scatters broadly around this well-known trend. Structural analysis [Fig. 1(c)] shows that most materials exhibit the tendency to contract vertically upon reduction (up to $50 \%$ ) with respect to their cubic phase, expanding simultaneously in the plane (up to $10 \%$ ) with reduced volume, particularly those materials where the reaction is exothermic $\left(E_{f}^{V_{\mathrm{O}}}<0\right)$. For some very stable and difficult to reduce compounds (i.e., featuring a high $E_{f}^{V_{\mathrm{O}}}$ ), the changes are rather modest (center of the plot; e.g., $\mathrm{BaHfO}_{n}$ and $\mathrm{LaAlO}_{n}$, Table I). In sharp contrast, a few materials expand massively in apical direction $\left(c_{0}^{\mathrm{IL}} / a_{0}^{\mathrm{IL}} \sim 2-3\right)$ with $10-20 \%$ basal contraction relative to the $\mathrm{P}$ phase. $\mathrm{PbTiO}_{n}$ is an example for such a basal contraction and concomitant apical expansion (Table I), which distinguishes it from all other listed materials [53].

Figure 1(a) places the formally $d^{9}$ IL nickelates and cuprates in an interesting context (cf. Table I). The nickelates appear as a compact family in the phase diagram, exhibiting a stable $\mathrm{P}$ phase, but being simultaneously close to the IL regime; the related palladates [33] and platinates are even more easily reduced. In contrast, the cuprate family extends widely over the IL region. This reflects the naturally preferred fourfold coordinated plaquette structure typical for high- $T_{C}$ cuprate superconductors. The $\mathrm{SrNiO}_{n}$ values show how Sr doping affects the rare-earth nickelates [23,29,35,38], destabilizing the $\mathrm{P}$ structure from $\sim-13$ to $-9.9 \mathrm{eV}$ and concomitantly facilitating their reduction to the IL geometry. Continuing the formal $d^{9}$ series, alkali-metal $\mathrm{Zn} / \mathrm{Cd} / \mathrm{Hg}$ oxides emerge, being again more compact and located deeper within the IL regime. Further interesting compounds can be identified in the IL region that simultaneously exhibit a highly 
TABLE I. Energies and lattice parameters for a selection of oxides (cf. Fig. 1). $E_{f}^{V_{O}}<0$ indicates that the IL structure $(n=2)$ is preferred over the the $\mathrm{P}$ phase $(n=3)$.

\begin{tabular}{lccccc}
\hline \hline System & $E_{f}^{V_{\mathrm{O}}}(\mathrm{eV})$ & $E_{f}^{\mathrm{P}}(\mathrm{eV})$ & $a_{0}^{\mathrm{P}}(\AA)$ & $a_{0}^{\mathrm{IL}}(\AA)$ & $c_{0}^{\mathrm{IL}}(\AA)$ \\
\hline $\mathrm{LaNiO}_{n}$ & 2.8 & -13.2 & 3.82 & 3.93 & 3.40 \\
$\mathrm{PrNiO}_{n}$ & 2.7 & -12.8 & 3.80 & 3.91 & 3.35 \\
$\mathrm{NdNiO}_{n}$ & 2.7 & -12.8 & 3.79 & 3.90 & 3.30 \\
$\mathrm{LuNiO}_{n}$ & 2.1 & -12.2 & 3.70 & 3.81 & 3.02 \\
$\mathrm{SrNiO}_{n}$ & 0.5 & -9.9 & 3.83 & 3.86 & 3.53 \\
$\mathrm{LaPdO}_{n}$ & 1.6 & -10.8 & 4.04 & 4.20 & 3.32 \\
$\mathrm{NdPdO}_{n}$ & 1.4 & -10.1 & 4.02 & 4.18 & 3.24 \\
$\mathrm{CaCuO}_{n}$ & -0.01 & -8.5 & 3.80 & 3.87 & 3.21 \\
$\mathrm{SrCuO}_{n}$ & 0.44 & -8.7 & 3.89 & 3.95 & 3.49 \\
$\mathrm{LiZnO}_{n}$ & -1.6 & -3.9 & 3.86 & 3.79 & 3.08 \\
$\mathrm{NaZnO}_{n}$ & -1.2 & -4.3 & 3.91 & 3.85 & 3.41 \\
$\mathrm{LaLiO}_{n}$ & -0.5 & -11.7 & 3.80 & 4.01 & 3.26 \\
$\mathrm{LaNaO}_{n}$ & -1.9 & -8.8 & 4.06 & 4.27 & 3.23 \\
\hline $\mathrm{BaHfO}_{n}$ & 6.8 & -18.9 & 4.20 & 4.21 & 4.05 \\
$\mathrm{SrTiO}_{n}$ & 5.8 & -17.7 & 3.94 & 3.99 & 3.59 \\
$\mathrm{LaAlO}_{n}$ & 6.7 & -18.6 & 3.81 & 3.83 & 3.67 \\
$\mathrm{NdGaO}_{n}$ & 5.2 & -14.7 & 3.88 & 4.09 & 3.34 \\
$\mathrm{LaTiO}_{n}$ & 5.6 & -18.0 & 3.96 & 4.01 & 3.55 \\
$\mathrm{PbTiO}_{n}$ & 5.0 & -13.4 & 3.97 & 3.92 & 4.08 \\
$\mathrm{CaSiO}_{n}$ & 6.2 & -16.0 & 3.61 & 3.80 & 3.22 \\
\hline \hline
\end{tabular}

negative $E_{f}^{\mathrm{P}}$. Exemplarily, $\mathrm{LaLiO}_{n}$ and $\mathrm{LaNaO}_{n}$ emerge as strongly anisotropic IL structures [Fig. 1(a), Table I]. They are insulators due to an $A^{3+} B^{1+} \mathrm{O}_{2}^{2-}$ configuration and thus may serve as quantum confinement layers.

The results listed in Table I are in close agreement with the literature, e.g., $\mathrm{NdNiO}_{2}\left(a_{0}^{\mathrm{IL}}=3.92, c_{0}^{\mathrm{IL}}=3.28 \AA\right.$ $[24,54]), \mathrm{PrNiO}_{2}\left(3.92,3.30 \AA\right.$ [39]), $\mathrm{LaNiO}_{2}(3.96,3.37 \AA$ $[28,55]), \mathrm{SrCuO}_{2}(3.93,3.43 \AA[56,57]), \mathrm{CaCuO}_{2} \quad(3.85$, $3.18 \AA[56,57])$; cubic $\mathrm{SrTiO}_{3}\left(a_{0}^{\mathrm{P}}=3.94 \AA\right.$ [58,59] $)$ and $\mathrm{PbTiO}_{3}\left(a_{0}^{\mathrm{P}}=3.97 \AA\right.$ [58] $)$; and pseudocubic $\mathrm{LaNiO}_{3}\left(a_{0}^{\mathrm{P}}=\right.$ $3.84 \AA[60,61])$. The layer reduction energies $E_{f}^{V_{\mathrm{O}}}$ can be related to oxygen vacancy formation energies. For $\mathrm{SrTiO}_{3}$, the formal $\mathrm{Ti}^{4+\rightarrow 2+}$ valence change renders the IL structure unattractive, in line with an oxygen vacancy formation energy of $\sim 5.5 \mathrm{eV}$ in bulk $\mathrm{SrTiO}_{3}$ [62]. For $\mathrm{LaAlO}_{3}$, this energy is even higher (6.9 eV [63]). The values in nickelates and their heterostructures [34,64] are much lower $(\sim 2.8 \mathrm{eV}$ in bulk $\mathrm{LaNiO}_{3}$ [65]), perfectly aligning with Table I.

Figures 2(a) and 2(b) show averaged $\left\langle E_{f}^{V_{\mathrm{O}}}\right\rangle$ for either a fixed $A$ or $B$ site, respectively, unraveling site- and elementresolved trends in the relative stability of $\mathrm{P}$ and IL phases across the periodic table. At the $A$ site, most of the central transition metals induce strong tendencies towards the planar IL configuration, particularly $\mathrm{W}$. The remaining elements generally stabilize the $\mathrm{P}$ structure, specifically $\mathrm{Ca}, \mathrm{Sr}, \mathrm{Ba}, \mathrm{Sc}$, $\mathrm{Y}, \mathrm{Pb}$, and the rare-earth metals. We observe a decreasing trend of $\left\langle E_{f}^{V_{\mathrm{O}}}\right\rangle$ across the rare-earth metals from 3.2 (La) to $2.2 \mathrm{eV}(\mathrm{Lu})$, and shifting from the Sc group (including rare-earth metals, $\left.A^{3+}\right)$ to the alkali metals $\left(A^{1+}\right)$. For instance, exchanging $\mathrm{La}$ with $\mathrm{Sr}$ facilitates the reduction by $0.8 \mathrm{eV}$ on average [62,66], and by additional $\sim 1.5 \mathrm{eV}$ if $A$ is an alkali metal. The $B$ site exhibits a much higher contrast among the different elements, $\left\langle E_{f}^{V_{\mathrm{O}}}\right\rangle$ ranging from -4.5 to $4.3 \mathrm{eV}$. Alkali metals, particularly $\mathrm{K}$, induce the IL phase. The late transition metals ( $\mathrm{Ni}, \mathrm{Cu}, \mathrm{Zn}$ groups) largely display increasingly negative $\left\langle E_{f}^{V_{\mathrm{O}}}\right\rangle$ values as well, which highlights their tendency towards the planar IL geometry discussed above [Fig. 1(a)]. In contrast, the $\mathrm{P}$ phase is clearly preferred by the technologically important early transition metals (even more for the $5 d$ than for the $3 d$ elements) as well as by the aluminates. Interestingly, also $\mathrm{Si}$ favors the formation of $\mathrm{P}$ oxides such as $\mathrm{Mg}^{2+} \mathrm{Si}^{4+} \mathrm{O}_{3}^{2-}$ and $\mathrm{Ca}^{2+} \mathrm{Si}^{4+} \mathrm{O}_{3}^{2-}$, which are indeed abundant in the lower part of the Earth's mantle [67].

The symmetric matrix in Fig. 2(c) displays the Pearson product-moment correlation coefficients $r_{i j}$ between different observables $x_{i}$, the latter ranging from atomic properties of the $A$ - and $B$-site elements to the energies and lattice parameters as determined from first principles. Here, $r_{i j}=c_{i j} / \sqrt{c_{i i} c_{j j}}$ with the covariance matrix $c_{i j}=\left\langle\left(x_{i}-\left\langle x_{i}\right\rangle\right)\left(x_{j}-\left\langle x_{j}\right\rangle\right)\right\rangle$, where averaging is performed over the entire database of 4692 materials. $E_{f}^{\mathrm{P}}$ shows a modest dependence on the $A$ site, whereas $E_{f}^{V_{\mathrm{O}}}$ lacks significant correlations apart from being anticorrelated with $E_{f}^{\mathrm{P}}(-0.8)$, which reflects the linear trend observed in Fig. 1. $a_{0}^{\mathrm{P}}$ and $a_{0}^{\mathrm{IL}}$ correlate predominantly with the $B$ site, particularly $r_{B}(0.8)$, and are also significantly
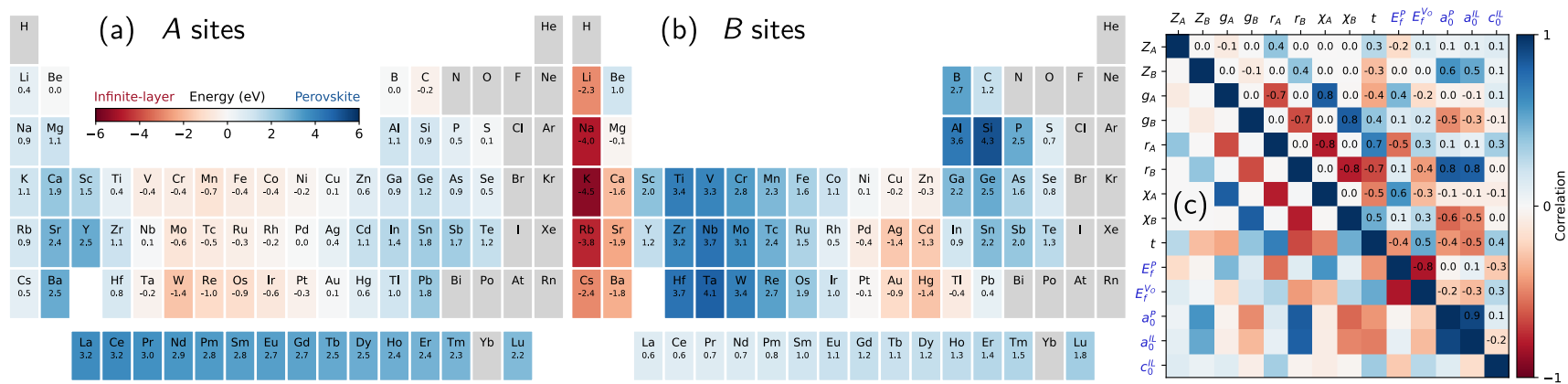

FIG. 2. Statistical analysis of IL and P oxides. Panels (a) and (b) display trends of $\left\langle E_{f}^{V_{\mathrm{O}}}\right\rangle$ (in color and explicit values) across the periodic table, fixing either the $A$ or the $B$ site and subsequently averaging over the other site, indicating site-resolved which elements tend to stabilize which of the two phases. Elements marked in grey were not considered. (c) The correlation matrix $r_{i j}$ unravels the interdependence of the different observables, including atomic properties (atomic number $Z$, periodic table group $g$, atomic radius $r$, and electronegativity $\chi$ ), the Goldschmidt tolerance factor $t$, and different energies and lattice parameters as determined from first principles (blue labels). 
intercorrelated (0.9). In sharp contrast, $c_{0}^{\mathrm{IL}}$ exhibits almost no correlations with the other quantities, at most with the Goldschmidt tolerance factor $t(0.4)$. While optimized descriptors $[6,7,9]$ may enhance the correlation, this indicates that a nonlinear methodology is required to reliably predict this quantity, which turns out to be challenging, as shown below.

\section{ACTIVE LEARNING OF NEURAL NETWORKS}

The interesting question arises whether the insights presented so far would have been possible without explicitly calculating the entire data set, but only a fraction of it. The challenge consists in finding a small but optimal subset of the materials parameter space that contains a maximum of non-redundant information, which is subsequently used by NNs to accurately predict the properties of all materials in the parameter space. We address this aspect by implementing an active learning (AL) algorithm, which constitutes a form of semisupervised learning $[2,42]$ and is described in the following.

Two NNs are trained in parallel [Fig. 3(a)]. They take the names of the elements at the $A$ and $B$ sites as categorical input, which are one-hot encoded and subsequently processed by a 16-dimensional embedding layer [17]. Such element embedding is inspired by word embedding [41], a technique used in language processing to represent words in a semantically insightful way in a vector space of compact dimension. In the present context of materials physics, embedding autonomously establishes a new and efficient representation of the high-dimensional one-hot-encoding vectors that describe the materials composition, i.e., it maps each of them onto a new vector in the embedding space. While this is at first sight only a (nonlinear) mathematical transformation, we will analyze below that the established representation unambiguously encodes information about the elements' chemical properties. Note that the norm of the embedding vectors also encodes information, owing to the nonlinearity of the NNs. Optionally, the NNs feature a parallel numerical input channel to complement the output of the embedding layer by the atomic radii $r_{A, B}$ and the electronegativities $\chi_{A, B}$, which turned out to be largely redundant in view of the more powerful embedding technique. This input layer is followed by a sequence of hidden layers, featuring 512, 256, and 128 densely connected neurons, respectively. We explored different $\mathrm{NN}$ architectures and found the present one to yield optimal results. The output layer provides energies $\left(E_{f}^{V_{\mathrm{o}}}, E_{f}^{\mathrm{P}}, E_{f}^{\mathrm{IL}}\right)$ or lattice parameters $\left(a_{0}^{\mathrm{P}}, a_{0}^{\mathrm{IL}}, c_{0}^{\mathrm{IL}}\right)$. We apply error backpropagation on the training set (a small subset of the parameter space, here initially $\sim 20 \%$ ) to automatically adapt the weights that connect the individual neurons, until an optimal mapping from input to output is achieved.

At this stage, the question is how to optimally increase the training set size to enhance the prediction accuracy [68]. Here, we compare the individual predictions by the two NNs to estimate which materials are attractive candidates: Given the observables $x_{i}^{1,2}$ as predicted by NN 1 and NN 2 and the respective DFT ground truth $x_{i}^{\mathrm{DFT}}$ (where $i$ labels either different energies or lattice parameters), we define by averaging

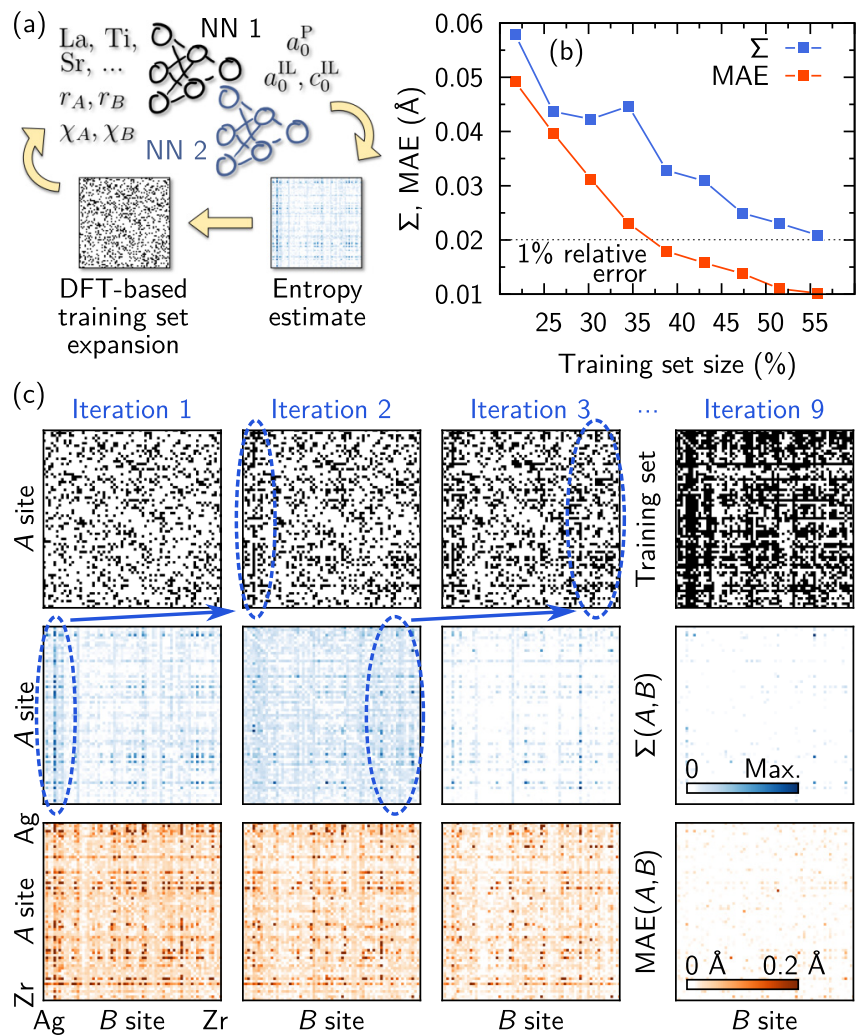

FIG. 3. (a) Active learning (AL) cycle of element-embedding neural networks. (b) Evolution of $\Sigma$ and the MAE (see text) with the number of AL iterations, shown exemplarily for lattice constant prediction. (c) Parameter space maps monitor consecutive AL iterations, the chemical elements being ordered alphabetically (each pixel in the matrix-like plots represents a distinct $A-B$ combination). For $\Sigma$ (blue, middle row), the color scale adaptively maximizes the contrast in each case, highlighting those materials that are selected by the algorithm for updating the training set. This training set is shown in the top row, where black (white) pixels mark $A-B$ combinations that are (not) included. The MAE (red, bottom row) is not used during $\mathrm{AL}$, but can be exploited a posteriori to trace the performance.

over $i$ :

$$
\begin{aligned}
\operatorname{MAE}(A, B) & =\left\langle\left|\frac{x_{i}^{1}(A, B)+x_{i}^{2}(A, B)}{2}-x_{i}^{\mathrm{DFT}}(A, B)\right|\right\rangle_{i} \\
\Sigma(A, B) & =\left\langle\mid x_{i}^{1}(A, B)-x_{i}^{2}(A, B) \|\right\rangle_{i}
\end{aligned}
$$

Site averaging yields the mean absolute error MAE $=$ $\langle\operatorname{MAE}(A, B)\rangle_{A, B}$ and $\Sigma=\langle\Sigma(A, B)\rangle_{A, B}$. In the AL cycle [Fig. 3(a)], the training set is now updated iteratively, appending 200 materials per step that exhibit the highest $\Sigma(A, B)$, followed by further $\mathrm{NN}$ training. Interestingly, this quantity represents an estimate of the local entropy in the parameter space, which would read $H(A, B) \sim \sum_{i} \log \sigma_{i}(A, B)$ in case the predictions $x_{i}$ followed uncorrelated normal distributions with $\sigma_{i}(A, B) \sim\left|x_{i}^{1}(A, B)-x_{i}^{2}(A, B)\right|$. In this spirit, the present AL algorithm statistically maximizes the information entailed in the training set. From the definition of $\Sigma(A, B)$ it follows that the DFT ground truth beyond the current training set is not required by the AL algorithm to select interesting 

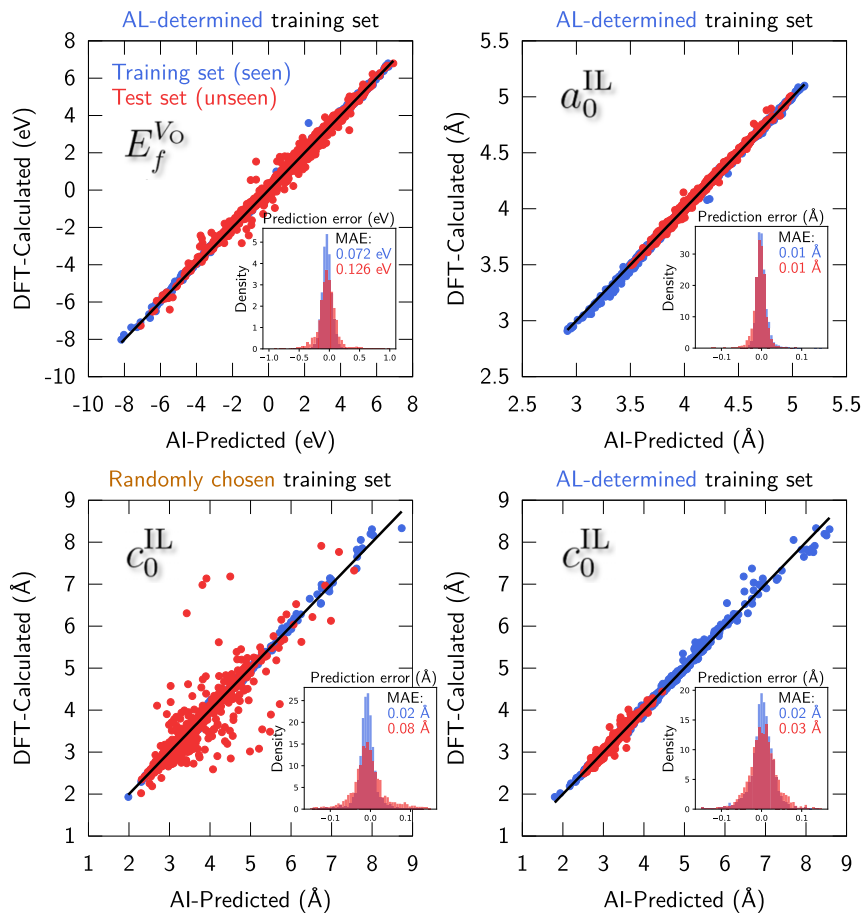

FIG. 4. Prediction of $E_{f}^{V_{\mathrm{O}}}, a_{0}^{\mathrm{IL}}$, and $c_{0}^{\mathrm{IL}}$ by a single NN versus the DFT ground truth, using only $\sim 50 \%$ of the data as training set (blue points, seen by the NN). The red points represent the test set, which has never been presented to the $\mathrm{NN}$ before. Contrasting the AL results with those obtained for a randomly chosen training set of equal size reveals the advantages of AL. The predictions for $a_{0}^{\mathrm{P}}$ and heats of formation $E_{f}^{\mathrm{P}}, E_{f}^{\mathrm{IL}}$ are even more accurate (not shown).

materials candidates; we use it only a posteriori to analyze the AL performance [Figs. 3(b) and 3(c)].

Figure 4 provides an impression of the $\mathrm{NN}$ accuracy. The prediction of the lattice parameters $a_{0}^{\mathrm{P}}$ (not shown) and $a_{0}^{\mathrm{IL}}$ proved to be straightforward, whereas $c_{0}^{\mathrm{IL}}$ turned out to be challenging. This can be traced back to the sparse data available for vertically expanding materials [Fig. 1(c)] and the only weak correlations of $c_{0}^{\mathrm{IL}}$ with other observables [Fig. 2(c)]. Here, AL considerably enhances the prediction accuracy as compared to a randomly chosen training set (Fig. 4). As an example, boron at the $B$ site, combined with a post-transitionmetal element at the $A$ site, tends to induce a large vertical expansion. Already in the first iteration, these unconventional compounds are automatically identified and included in the training set [marked by the dashed ellipse in Fig. 3(c)].

AL-iterating towards $\sim 50 \%$ training set size, we already obtain a MAE $\sim 0.1 \mathrm{eV}$ for $E_{f}^{V_{\mathrm{o}}}$ per vacancy (Fig. 4). Relative to their overall range of $\sim 15 \mathrm{eV}$, this corresponds to an error of $<0.7 \%$. The heats of formation are predicted even more accurately, reaching $25 \mathrm{meV}$ /atom (not shown), which is comparable to recent work on perovskites $(20-34 \mathrm{meV} /$ atom [12]). This reflects that $E_{f}^{V_{0}}$ is a fingerprint of the complex reaction and thus more demanding to predict. For elpasolites, a heat of formation accuracy of $150 \mathrm{meV} /$ atom was obtained [17]. As a reference, the DFT accuracy can be considered as $\sim 0.1 \mathrm{eV}[11,69]$. A MAE of $0.2 \mathrm{eV}$ is achieved already around 35\% training set size. A similar trend can be seen for the lattice parameters [Fig. 3(b)]. In general, we observed that

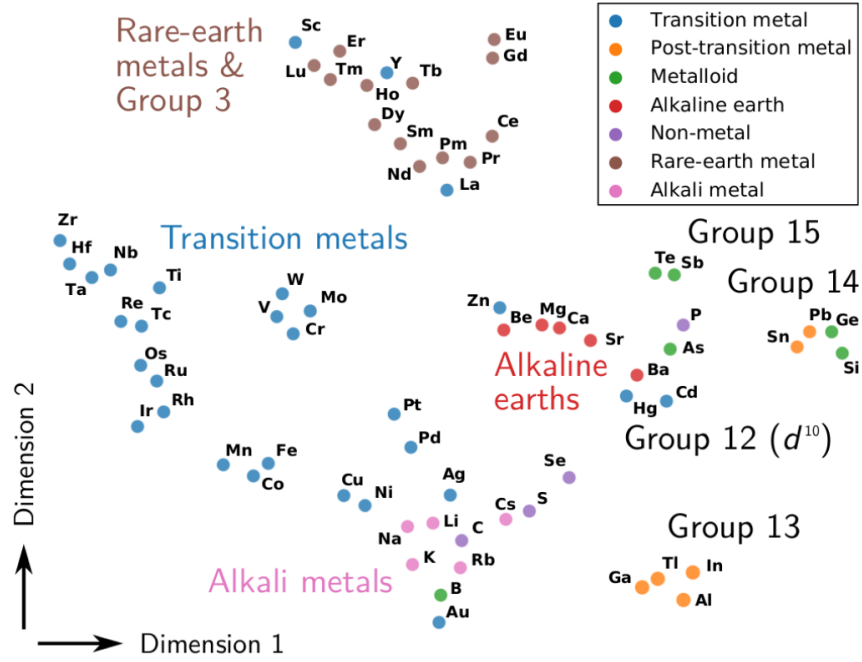

FIG. 5. Stochastic neighbor embedding ( $t$-SNE) analysis of the element embedding vectors (projection of 16 to 2 dimensions) shows that the NNs automatically develop their own concept of chemical similarity while being trained on the IL and P oxide data. Note the formation of distinct element clusters that correspond to different families and groups of the periodic table.

ensemble-averaged predictions of multiple NNs (here: two) are more accurate than predictions by the individual NNs, attaining $<1 \%$ relative error for $\sim 35 \%$ training set size.

Figure 5 explores the automatically generated $\mathrm{NN}$ element embedding vectors by using stochastic neighbor embedding $(t$-SNE [70]) as implemented in the scikit-learn library [71]. This technique represents the element vectors from the 16dimensional embedding space (see above) by points in two dimensions. To this end, it constructs a probability distribution over vector pairs in the embedding space using the Euclidean norm, defines a similar probability distribution over the points in the two-dimensional representation, and minimizes the Kullback-Leibler divergence between the two probability distributions with respect to the points' coordinates. The two dimensions are rather of mathematical character and do not necessarily have a physical correspondence. Despite the complexity of this technique, it reveals that the NNs develop a very unique (i.e., unbiased) understanding of the chemical similarity between the individual elements, mirroring the conventional picture of the periodic table. Specifically, several distinct clusters emerge, such as the rare-earth metals (interestingly also comprising the group- 3 elements $\mathrm{Sc}$ and $\mathrm{Y}$ ), the transition metals (the $d^{10}$ elements $\mathrm{Zn}, \mathrm{Cd}$, and $\mathrm{Hg}$ being slightly separated), the group-13 elements, and the group-14 elements. Alkali metals and alkaline earths are closely grouped as well, even though the $t$-SNE projection places them somewhat within the transition metal cluster. The group-16 elements Se and Te are more dispersed and reflect predominantly their nonmetal and metalloid classification. The identification of these chemical trends is even more compelling as the NNs are entirely agnostic about concepts such as the atomic number or the group of a particular element. In addition, we observed that this element-embedding approach increases the accuracy as compared to directly passing the high-dimensional one-hot encoded element vectors to the densely connected hidden layers. 
The presented $\mathrm{AL}$ algorithm can be stopped as soon as the desired accuracy is reached [Fig. 3(b)], establishing the latter as a systematic control parameter. Moreover, only the autonomously selected materials need to be calculated $a b$ initio in each iteration. These aspects lead to a substantial gain in performance and energy efficiency as compared to conventional high-throughput calculations. The presented methodology can be straightforwardly generalized to efficiently predict and enhance a broad scope of observables, e.g., the thermoelectric performance [72-74], across a large variety of interesting materials classes.

\section{ACKNOWLEDGMENTS}

We thank Professor Dr. R. Pentcheva (University of Duisburg-Essen), Professor Dr. M. Marques (University of Halle-Wittenberg), and Professor Dr. A. Ecker (University of Göttingen) for helpful discussions. B.G. acknowledges financial support provided by an Award in the Excellent Early Career Researchers Funding Competition of the University of Duisburg-Essen and a Grant by the Department of Physics of the University of Duisburg-Essen. We acknowledge support by the Open Access Publication Fund of the University of Duisburg-Essen.
[1] K. T. Butler, D. W. Davies, H. Cartwright, O. Isayev, and A. Walsh, Machine learning for molecular and materials science, Nature (London) 559, 547 (2018).

[2] J. Schmidt, M. R. G. Marques, S. Botti, and M. A. L. Marques, Recent advances and applications of machine learning in solidstate materials science, npj Comput. Mater. 5, 83 (2019).

[3] A. Y.-T. Wang, R. J. Murdock, S. K. Kauwe, A. O. Oliynyk, A. Gurlo, J. Brgoch, K. A. Persson, and T. D. Sparks, Machine learning for materials scientists: An introductory guide toward best practices, Chem. Mat. 32, 4954 (2020).

[4] L. Ward, A. Agrawal, A. Choudhary, and C. Wolverton, A general-purpose machine learning framework for predicting properties of inorganic materials, npj Comput. Mater. 2, 16028 (2016).

[5] F. A. Faber, A. Lindmaa, O. A. von Lilienfeld, and R. Armiento, Machine Learning Energies of 2 Million Elpasolite $\left(A B C_{2} D_{6}\right)$ Crystals, Phys. Rev. Lett. 117, 135502 (2016).

[6] L. M. Ghiringhelli, J. Vybiral, S. V. Levchenko, C. Draxl, and M. Scheffler, Big Data of Materials Science: Critical Role of the Descriptor, Phys. Rev. Lett. 114, 105503 (2015).

[7] C. J. Bartel, C. Sutton, B. R. Goldsmith, R. Ouyang, C. B. Musgrave, L. M. Ghiringhelli, and M. Scheffler, New tolerance factor to predict the stability of perovskite oxides and halides, Sci. Adv. 5, eaav0693 (2019).

[8] J. Schmidt, J. Shi, P. Borlido, L. Chen, S. Botti, and M. A. L. Marques, Predicting the thermodynamic stability of solids combining density functional theory and machine learning, Chem. Mat. 29, 5090 (2017).

[9] F. Belviso, V. E. P. Claerbout, A. Comas-Vives, N. S. Dalal, F.-R. Fan, A. Filippetti, V. Fiorentini, L. Foppa, C. Franchini, B. Geisler, L. M. Ghiringhelli, A. Groß, S. Hu, J. Íñiguez, S. K. Kauwe, J. L. Musfeldt, P. Nicolini, R. Pentcheva, T. Polcar, W. Ren et al., Viewpoint: Atomic-scale design protocols toward energy, electronic, catalysis, and sensing applications, Inorg. Chem. 58, 14939 (2019).

[10] A. Lopez-Bezanilla and P. B. Littlewood, Growing field of materials informatics: databases and artificial intelligence, MRS Commun. 10, 1 (2020).

[11] T. Xie and J. C. Grossman, Crystal Graph Convolutional Neural Networks for an Accurate and Interpretable Prediction of Material Properties, Phys. Rev. Lett. 120, 145301 (2018).

[12] W. Ye, C. Chen, Z. Wang, I.-H. Chu, and S. P. Ong, Deep neural networks for accurate predictions of crystal stability, Nat. Commun. 9, 3800 (2018).

[13] D. Jha, K. Choudhary, F. Tavazza, W.-k. Liao, A. Choudhary, C. Campbell, and A. Agrawal, Enhancing materials property prediction by leveraging computational and experimental data using deep transfer learning, Nat. Commun. 10, 5316 (2019).

[14] A. Agrawal and A. Choudhary, Deep materials informatics: Applications of deep learning in materials science, MRS Commun. 9, 779 (2019).

[15] L. Zhang, J. Han, H. Wang, R. Car, and W. E, Deep Potential Molecular Dynamics: A Scalable Model with the Accuracy of Quantum Mechanics, Phys. Rev. Lett. 120, 143001 (2018).

[16] V. Tshitoyan, J. Dagdelen, L. Weston, A. Dunn, Z. Rong, O. Kononova, K. A. Persson, G. Ceder, and A. Jain, Unsupervised word embeddings capture latent knowledge from materials science literature, Nature (London) 571, 95 (2019).

[17] Q. Zhou, P. Tang, S. Liu, J. Pan, Q. Yan, and S.-C. Zhang, Learning atoms for materials discovery, Proc. Natl. Acad. Sci. USA 115, E6411 (2018).

[18] D. Jha, L. Ward, A. Paul, W.-k. Liao, A. Choudhary, C. Wolverton, and A. Agrawal, ElemNet: Deep learning the chemistry of materials from only elemental composition, Sci. Rep. 8, 17593 (2018).

[19] A. Jain, S. P. Ong, G. Hautier, W. Chen, W. D. Richards, S. Dacek, S. Cholia, D. Gunter, D. Skinner, G. Ceder, and K. A. Persson, The Materials Project: A materials genome approach to accelerating materials innovation, APL Mater. 1, 011002 (2013).

[20] J. E. Saal, S. Kirklin, M. Aykol, B. Meredig, and C. Wolverton, Materials design and discovery with high-throughput density functional theory: The Open Quantum Materials Database (OQMD), JOM 65, 1501 (2013).

[21] S. Curtarolo, W. Setyawan, G. L. Hart, M. Jahnatek, R. V. Chepulskii, R. H. Taylor, S. Wang, J. Xue, K. Yang, O. Levy, M. J. Mehl, H. T. Stokes, D. O. Demchenko, and D. Morgan, AFLOW: An automatic framework for high-throughput materials discovery, Comp. Mat. Sci. 58, 218 (2012).

[22] C. Draxl and M. Scheffler, Nomad: The fair concept for big data-driven materials science, MRS Bull. 43, 676 (2018).

[23] D. Li, K. Lee, B. Y. Wang, M. Osada, S. Crossley, H. R. Lee, Y. Cui, Y. Hikita, and H. Y. Hwang, Superconductivity in an infinite-layer nickelate, Nature (London) 572, 624 (2019).

[24] Y. Nomura, M. Hirayama, T. Tadano, Y. Yoshimoto, K. Nakamura, and R. Arita, Formation of a two-dimensional single-component correlated electron system and band engineering in the nickelate superconductor $\mathrm{NdNiO}_{2}$, Phys. Rev. B 100, 205138 (2019).

[25] P. Jiang, L. Si, Z. Liao, and Z. Zhong, Electronic structure of rare-earth infinite-layer $R \mathrm{NiO}_{2}(R=\mathrm{La}, \mathrm{Nd})$, Phys. Rev. B 100, 201106(R) (2019). 
[26] H. Sakakibara, H. Usui, K. Suzuki, T. Kotani, H. Aoki, and K. Kuroki, Model Construction and a Possibility of Cupratelike Pairing in a New $d^{9}$ Nickelate Superconductor (Nd, $\left.\mathrm{Sr}\right) \mathrm{NiO}_{2}$, Phys. Rev. Lett. 125, 077003 (2020).

[27] M. Jiang, M. Berciu, and G. A. Sawatzky, Critical Nature of the $\mathrm{Ni}$ Spin State in Doped $\mathrm{NdNiO}_{2}$, Phys. Rev. Lett. 124, 207004 (2020).

[28] A. S. Botana and M. R. Norman, Similarities and differences between $\mathrm{LaNiO}_{2}$ and $\mathrm{CaCuO}_{2}$ and Implications for Superconductivity, Phys. Rev. X 10, 011024 (2020).

[29] M. Osada, B. Y. Wang, B. H. Goodge, K. Lee, H. Yoon, K. Sakuma, D. Li, M. Miura, L. F. Kourkoutis, and H. Y. Hwang, A superconducting praseodymium nickelate with infinite layer structure, Nano Lett. 20, 5735 (2020).

[30] F. Lechermann, Late transition metal oxides with infinitelayer structure: Nickelates versus cuprates, Phys. Rev. B 101, 081110(R) (2020).

[31] X. Wu, D. Di Sante, T. Schwemmer, W. Hanke, H. Y. Hwang, S. Raghu, and R. Thomale, Robust $d_{x^{2}-y^{2}}$-wave superconductivity of infinite-layer nickelates, Phys. Rev. B 101, 060504(R) (2020).

[32] M. Hirayama, T. Tadano, Y. Nomura, and R. Arita, Materials design of dynamically stable $d^{9}$ layered nickelates, Phys. Rev. B 101, 075107 (2020).

[33] M. Kitatani, L. Si, O. Janson, R. Arita, Z. Zhong, and K. Held, Nickelate superconductors - a renaissance of the oneband Hubbard model, npj Quantum Mater. 5, 59 (2020).

[34] B. Geisler and R. Pentcheva, Fundamental difference in the electronic reconstruction of infinite-layer versus perovskite neodymium nickelate films on $\mathrm{SrTiO}_{3}(001)$, Phys. Rev. B 102, 020502(R) (2020).

[35] D. Li, B. Y. Wang, K. Lee, S. P. Harvey, M. Osada, B. H. Goodge, L. F. Kourkoutis, and H. Y. Hwang, Superconducting Dome in $\mathrm{Nd}_{1-x} \mathrm{Sr}_{x} \mathrm{NiO}_{2}$ Infinite Layer Films, Phys. Rev. Lett. 125, 027001 (2020).

[36] M.-Y. Choi, K.-W. Lee, and W. E. Pickett, Role of $4 f$ states in infinite-layer $\mathrm{NdNiO}_{2}$, Phys. Rev. B 101, 020503(R) (2020).

[37] L. Si, W. Xiao, J. Kaufmann, J. M. Tomczak, Y. Lu, Z. Zhong, and K. Held, Topotactic Hydrogen in Nickelate Superconductors and Akin Infinite-Layer Oxides $A B \mathrm{O}_{2}$, Phys. Rev. Lett. 124, 166402 (2020).

[38] Y. Wang, C.-J. Kang, H. Miao, and G. Kotliar, Hund's metal physics: From $\mathrm{SrNiO}_{2}$ to $\mathrm{LaNiO}_{2}$, Phys. Rev. B 102, 161118(R) (2020).

[39] B. Geisler and R. Pentcheva, Correlated interface electron gas in infinite-layer nickelate versus cuprate films on $\mathrm{SrTiO}_{3}(001)$, Phys. Rev. Res. 3, 013261 (2021).

[40] R. A. Ortiz, H. Menke, F. Misják, D. T. Mantadakis, K. Fürsich, E. Schierle, G. Logvenov, U. Kaiser, B. Keimer, P. Hansmann, and E. Benckiser, Superlattice approach to doping infinite-layer nickelates, Phys. Rev. B 104, 165137 (2021).

[41] T. Mikolov, K. Chen, G. Corrado, and J. Dean, Efficient estimation of word representations in vector space (2013), arXiv:1301.3781.

[42] T. Lookman, P. V. Balachandran, D. Xue, and R. Yuan, Active learning in materials science with emphasis on adaptive sampling using uncertainties for targeted design, npj Comput. Mater. 5, 21 (2019).
[43] W. Kohn and L. J. Sham, Self-consistent equations including exchange and correlation effects, Phys. Rev. 140, A1133 (1965).

[44] G. Kresse and D. Joubert, From ultrasoft pseudopotentials to the projector augmented-wave method, Phys. Rev. B 59, 1758 (1999).

[45] P. E. Blöchl, Projector augmented-wave method, Phys. Rev. B 50, 17953 (1994).

[46] J. P. Perdew, K. Burke, and M. Ernzerhof, Generalized Gradient Approximation Made Simple, Phys. Rev. Lett. 77, 3865 (1996).

[47] We used an energy cutoff of $520 \mathrm{eV}$ and $U=3.32,3.7,5.3,3.9$, 4.38, 6.2, 3.25, and $6.2 \mathrm{eV}$ for $\mathrm{Co}, \mathrm{Cr}, \mathrm{Fe}, \mathrm{Mn}, \mathrm{Mo}, \mathrm{Ni}, \mathrm{V}$, and $\mathrm{W} d$ states, respectively. We ensured $k$-point convergence of the total energy within a few meV per unit cell (e.g., $12 \times 12 \times 12$ for $\mathrm{SrTiO}_{3}$ ), adjusting the $k$-point grid to the respective size of the Brillouin zone. This simultaneously renders accurate lattice parameters. Aspects of magnetism are not considered $[24,28,30,34]$. Rare-earth $4 f$ electrons were consistently frozen in the core [24,30,34,38,39,75].

[48] S. P. Ong, W. D. Richards, A. Jain, G. Hautier, M. Kocher, S Cholia, D. Gunter, V. L. Chevrier, K. A. Persson, and G. Ceder, Python materials genomics (pymatgen): A robust, open-source python library for materials analysis, Comp. Mat. Sci. 68, 314 (2013).

[49] A. I. Liechtenstein, V. I. Anisimov, and J. Zaanen, Densityfunctional theory and strong interactions: Orbital ordering in mott-hubbard insulators, Phys. Rev. B 52, R5467 (1995).

[50] M. Abadi, A. Agarwal, P. Barham, E. Brevdo, Z. Chen, C. Citro, G. S. Corrado, A. Davis, J. Dean, M. Devin, S. Ghemawat, I. Goodfellow, A. Harp, G. Irving, M. Isard, Y. Jia, R. Jozefowicz, L. Kaiser, M. Kudlur, J. Levenberg, et al., TensorFlow: Large-scale machine learning on heterogeneous systems (2015), software available from tensorflow.org.

[51] F. Chollet, Keras, https://keras.io (2015).

[52] The well-known overbinding of gas-phase $\mathrm{O}_{2}$ molecules in DFT necessitates a correction of $E\left(\mathrm{O}_{2}\right)$, which we performed such as to reproduce the experimental $\mathrm{O}_{2}$ binding energy of $5.16 \mathrm{eV}$ [64-66].

[53] This holds for the cubic paraelectric phase; relative to the tetragonal ferroelectric phase $(a=3.84, c=4.77 \AA$ [58]), which is $205 \mathrm{meV} /$ formula unit lower in energy, the IL geometry features basal expansion and vertical contraction.

[54] M. Hayward and M. Rosseinsky, Synthesis of the infinite layer $\mathrm{Ni}(\mathrm{I})$ phase $\mathrm{NdNiO}_{2+x}$ by low temperature reduction of $\mathrm{NdNiO}_{3}$ with sodium hydride, Solid State Sci. 5, 839 (2003).

[55] M. A. Hayward, M. A. Green, M. J. Rosseinsky, and J. Sloan, Sodium hydride as a powerful reducing agent for topotactic oxide deintercalation: Synthesis and characterization of the Nickel(I) oxide $\mathrm{LaNiO}_{2}$, J. Am. Chem. Soc. 121, 8843 (1999).

[56] N. Kobayashi, Z. Hiroi, and M. Takano, Compounds and phase relations in the $\mathrm{SrO}-\mathrm{CaO}-\mathrm{CuO}$ system under high pressure, J. Solid State Chem. 132, 274 (1997).

[57] Z. Zhong, G. Koster, and P. J. Kelly, Prediction of thickness limits of ideal polar ultrathin films, Phys. Rev. B 85, 121411(R) (2012).

[58] Y. Zhang, J. Sun, J. P. Perdew, and X. Wu, Comparative first-principles studies of prototypical ferroelectric materials by LDA, GGA, and SCAN meta-GGA, Phys. Rev. B 96, 035143 (2017). 
[59] V. Begum, M. E. Gruner, and R. Pentcheva, Role of the exchange-correlation functional on the structural, electronic, and optical properties of cubic and tetragonal $\mathrm{SrTiO}_{3}$ including many-body effects, Phys. Rev. Mater. 3, 065004 (2019).

[60] J. L. García-Muñoz, J. Rodríguez-Carvajal, P. Lacorre, and J. B. Torrance, Neutron-diffraction study of $R \mathrm{NiO}_{3}(R=$ $\mathrm{La}, \mathrm{Pr}, \mathrm{Nd}, \mathrm{Sm}$ ): Electronically induced structural changes across the metal-insulator transition, Phys. Rev. B 46, 4414 (1992).

[61] S. J. May, J.-W. Kim, J. M. Rondinelli, E. Karapetrova, N. A. Spaldin, A. Bhattacharya, and P. J. Ryan, Quantifying octahedral rotations in strained perovskite oxide films, Phys. Rev. B 82, 014110 (2010).

[62] M. T. Curnan and J. R. Kitchin, Effects of concentration, crystal structure, magnetism, and electronic structure method on first-principles oxygen vacancy formation energy trends in perovskites, J. Phys. Chem. C 118, 28776 (2014).

[63] C. Mitra, C. Lin, J. Robertson, and A. A. Demkov, Electronic structure of oxygen vacancies in $\mathrm{SrTiO}_{3}$ and $\mathrm{LaAlO}_{3}$, Phys. Rev. B 86, 155105 (2012).

[64] B. Geisler and R. Pentcheva, Inducing $n$ - and $p$-Type Thermoelectricity in Oxide Superlattices by Strain Tuning of Orbital-Selective Transport Resonances, Phys. Rev. Appl. 11, 044047 (2019).

[65] A. Malashevich and S. Ismail-Beigi, First-principles study of oxygen-deficient $\mathrm{LaNiO}_{3}$ structures, Phys. Rev. B 92, 144102 (2015).

[66] B. Geisler and R. Pentcheva, Competition of defect ordering and site disproportionation in strained $\mathrm{LaCoO}_{3}$ on $\mathrm{SrTiO}_{3}(001)$, Phys. Rev. B 101, 165108 (2020).

[67] F. Nestola, N. Korolev, M. Kopylova, N. Rotiroti, D. G. Pearson, M. G. Pamato, M. Alvaro, L. Peruzzo, J. J. Gurney, A. E. Moore, and J. Davidson, $\mathrm{CaSiO}_{3}$ perovskite in diamond indicates the recycling of oceanic crust into the lower mantle, Nature (London) 555, 237 (2018).

[68] In this work, we calculated the data for all materials in advance. If this were not the case, the data would be generated on the fly by DFT exclusively for those materials that are requested by the active-learning algorithm.

[69] S. Kirklin, J. E. Saal, B. Meredig, A. Thompson, J. W. Doak, M. Aykol, S. Rühl, and C. Wolverton, The Open Quantum Materials Database (OQMD): assessing the accuracy of DFT formation energies, npj Comput. Mater. 1, 15010 (2015).

[70] L. van der Maaten and G. Hinton, Visualizing data using $t$-SNE, J. Mach. Learn. Res. 9, 2579 (2008).

[71] F. Pedregosa, G. Varoquaux, A. Gramfort, V. Michel, B. Thirion, O. Grisel, M. Blondel, P. Prettenhofer, R. Weiss, V. Dubourg, J. Vanderplas, A. Passos, D. Cournapeau, M. Brucher, M. Perrot, and E. Duchesnay, Scikit-learn: Machine learning in Python, J. Mach. Learn. Res. 12, 2825 (2011).

[72] B. Geisler, A. Blanca-Romero, and R. Pentcheva, Design of $n$ - and $p$-type oxide thermoelectrics in $\mathrm{LaNiO}_{3} / \mathrm{SrTiO}_{3}(001)$ superlattices exploiting interface polarity, Phys. Rev. B 95, 125301 (2017).

[73] B. Geisler and R. Pentcheva, Confinement- and straininduced enhancement of thermoelectric properties in $\mathrm{LaNiO}_{3} / \mathrm{LaAlO}_{3}(001)$ superlattices, Phys. Rev. Mater. 2, 055403 (2018).

[74] G. Xing, J. Sun, Y. Li, X. Fan, W. Zheng, and D. J. Singh, Electronic fitness function for screening semiconductors as thermoelectric materials, Phys. Rev. Mater. 1, 065405 (2017).

[75] J. Liu, M. Kargarian, M. Kareev, B. Gray, P. J. Ryan, A. Cruz, N. Tahir, Y.-D. Chuang, J. Guo, J. M. Rondinelli, J. W. Freeland, G. A. Fiete, and J. Chakhalian, Heterointerface engineered electronic and magnetic phases of $\mathrm{NdNiO}_{3}$ thin films, Nat. Commun. 4, 2714 (2013). 